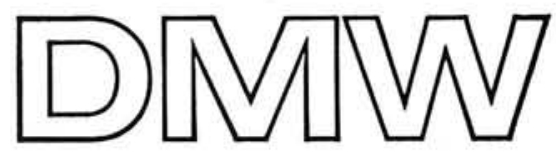

\title{
Feinnadelpunktion der Schilddrüse: sonographisch zielen
}

Der Beitrag von Baum und Mitarbeitern (1) in dieser Nummer (S. 1359) stellt die einzelnen Schritte der heutigen Diagnostik bei Schilddrüsenvergrößerungen oder Schilddrüsenknoten in eindrucksvoller Weise zusammen. $\mathrm{Zu}$ unterstreichen ist die Forderung, alle vergrößerten oder palpatorisch auffälligen Schilddrüsen sonographisch zu untersuchen, um diffuse oder herdförmige Abweichungen der Echostruktur zu erfassen. Die Sonographie ist bei der Erfassung von Knoten weit sensibler als die Palpation und Szintigraphie. Sie eignet sich ferner hervorragend zur objektiven Volumetrie und zur volumetrischen Verlaufsbeobachtung bei verschiedenen Schilddrüsenerkrankungen $(1,5,6)$. Die heutige Einteilung der Echostruktur in normal, echoreich, echoarm, echofrei und komplex ist eindeutig und beschreibt ohne Neigung zur Überinterpretation, die leider allen bildgebenden Verfahren anhaftet, alle möglichen, morphologisch verschiedenartigen Strukturvarianten.

Homogene, echonormale Schilddrüsenstrukturen bedürfen im allgemeinen keiner weiteren szintigraphischen und zytologischen Klärung, soweit keine abnormen Blutwerte, zum Beispiel Antikörpertiter oder Schilddrüsenhormonwerte, vorliegen. Alle Strukturabweichungen, besonders aber echoarme, echofreie und echokomplexe Befunde sollten durch die komplikationslose Feinnadelpunktion weiter untersucht werden, deren großzügiger Einsatz seit langem gefordert wird $(3,4)$. Baum und Mitarbeiter betonen die Notwendigkeit der gezielten Feinnadelbiopsie. Die diagnostische Treffsicherheit kann damit erheblich gesteigert werden. Sonographische Untersuchung und Punktion sollten aber von demselben Arzt durchgeführt werden, der gleichzeitig über eine adäquate Ausstrichtechnik verfügt.

Die Punktion erfolgt mit einer 10-ml-Einmalspritze und einer Einmalnadel (äußerer Durchmesser 0,6-0,9 $\mathrm{mm})$. Nach Punktion des verdächtigen Areals wird der Spritzenkolben maximal herausgezogen. Mittels fächerförmiger Bewegung erhält man Zellmaterial aus dem gesamten Bezirk. Vor dem Herausziehen der Nadel muß

Dtsch. med. Wschr. 108 (1983), 1343-1344

(C) 1983 Georg Thieme Verlag Stuttgart - New York der Spritzenkolben wieder freigegeben werden. Dadurch läßt sich die Aspiration von Blut, Fett oder von Hautbestandteilen, welche die Beurteilung stören, vermeiden. Dann wird die Spritze von der Nadel getrennt und mit Luft gefüllt. Mit der luftgefüllten Spritze läßt sich das Punktat vorsichtig als Tropfen auf einen Objektträger und je nach Konsistenz entweder wie ein Bluttropfen ausstreichen oder durch vorsichtigen Druck mit einem flach aufgelegten Objektträger verteilen. Das Vorlegen einer Kochsalzlösung in der Aspirationsspritze und das dann erforderliche Abzentrifugieren von Punktionsmaterial sind nicht zweckmäßig, da damit wertvolle Beurteilungskriterien wie Hintergrund (Kolloidmenge, Kolloidverteilung, Eiweißniederschläge, Blutbestandteile), Zellzahl und Zellanordnung verlorengehen. Lediglich das Präzipitat von abzentrifugierter Zystenflüssigkeit sollte gegebenenfalls sorgfältig auf Tumorzellen untersucht werden. Besser punktiert man nach Entleerung der Zyste das benachbarte Gewebe noch einmal oder schließt eine Kontrollpunktion wenige Tage später an.

Die von Baum und Mitarbeitern vorgenommene Befunderhebung nach einem modifizierten Papanicolaou-Schema halten wir für weniger glücklich. Sie erlaubt unnötige Unschärfen. PAP III beschreibt unklare, zweifelhafte Befunde, PAP IV wenige atypische Zellen oder Zellverbände. Der geübte Schilddrüsenzytologe ist dagegen mit über $90 \%$ iger Treffsicherheit in der Lage, in folgenden Fälle eine klare Diagnose zu stellen: gutartige Struma, Thyreoiditis (akut, subakut, chronisch), maligne Neoplasie (medullär, anaplastisch, malignes Lymphom, Metastasen). Häufigste Fehlerquellen sind mangelhafte Materialgewinnung und die Unerfahrenheit des Zytologen. Trotz verschiedener Ansätze gelingt es bis heute aber nicht, hochdifferenzierte follikuläre und papilläre Karzinome von entsprechenden Adenomen zu unterscheiden. Selbst dem Histologen fällt eine Unterscheidung oft schwer. Kapsel- und Gefäßeinbrüche bleiben dem Zytologen verborgen. Das zytologische Zellbild wird in diesen Fällen als follikuläre bzw. papilläre Neoplasie oder Proliferation beschrieben. Dasselbe gilt auch für die seltenen Onkozytome $(3,4)$. Eine histologische Klärung sollte in diesen Fällen immer angestrebt werden. 
Die Szintigraphie halten wir für indiziert zur Diagnostik der Schilddrüsenautonomie und zur Klärung sonographisch verdächtiger Knoten bei negativem Zytologiebefund. Sonographisch echokomplexe oder echoarme Knoten, die zytologisch unauffällig sind, sollten szintigraphisch geklärt werden. Ist das sonographisch verdächtige Areal szintigraphisch kalt, so sollte trotz negativen Zytologiebefundes eine histologische Klärung folgen, da Baum und Mitarbeiter angeben, daß sich die Wahrscheinlichkeit, ein Malignom anzutreffen, um den Faktor 3 auf etwa $25 \%$ erhöht, wenn ein sonographisch echoarmer oder echokomplexer Knoten gleichzeitig szintigraphisch kalt ist.

Übereinstimmend mit Baum und Mitarbeitern halten wir die Sonographie in Verbindung mit der Punktionszytologie - die Szintigraphie benötigt man vor allem für den Nachweis der Autonomie, aber auch kalter Knoten für eine hervorragende Möglichkeit zur Klärung herdförmiger, morphologischer Schilddrüsenveränderungen. Nur die Verbindung aller drei Methoden kann die Häufigkeit unnötiger Schilddrüsenoperationen herabsetzen.
Die höchste Treffsicherheit ist nur gewährleistet, wenn der punktierende Arzt auch gleichzeitig der sonographierende ist.

\section{Literatur}

(1) Baum, K., Chr. Reiners, W. Wiedemann, H.-A. Müller, W. Börner: Differentialdiagnose von Schilddrüsenknoten. Sonographie als Ergänzung der Szintigraphie und Punktionszytologie. Dtsch. med. Wschr. 108 (1983), 1359.

(2) Brunn, J. U., U. Block, G. Ruf, I. Bos, W. P. Kunze, P. C. Scriba: Volumetric der Schilddrüsenlappen mittels Real-TimeSonographie. Dtsch. med. Wschr. 106 (1981), 1338.

(3) Droese, M.: Aspirationszytologie der Schilddrüse (Schattauer: Stuttgart-New York 1979), 3.

\author{
(4) Löwhagen, T., J. S. Willems, G. Lun- \\ del, R. Sundblatt, P. O. Granberg: Aspi- \\ ration biopsy-cytology in diagnosis of \\ thyroid cancer. World J. Surg. 5 (1981), \\ 61. \\ (5) Olbricht, T., T. Schmitka, U. Melling \\ hoff, G. Benker, D. Reinwein: Sonogra- \\ phische Bestimmung von Schilddrüsenvo- \\ lumina bei Schilddrüsengesunden. Dtsch. \\ med. Wschr. 108 (1983), 1355. \\ (6) Scriba, P. C., C. Schneider, P. Pfan- \\ nenstiel, H. G. Heinze: Bildgebende Ver- \\ fahren in der Schilddrüsendiagnostik. \\ Akt. Endokr. 4, Suppl. 1 (1983), 1.
}

Dr. R. Gutekunst, Prof. Dr. P. C. Scriba

Klinik für Innere Medizin

Medizinische Hochschule

2400 Lübeck, Ratzeburger Allee 160 